\title{
جامعات عربية في بلدان أجنبية
}

\section{Arabic Universities abroad}

خالا مصطفى Khaled Moustafa Editor of Arabic Science Archive محرر الأرشيف العربي العلمي Email: khaled.moustafa@arabixiv.org

التطور العلمي والمعرفة الإنسانية هما حصيلة تفاعل وتراكم الأفكار والجهود الإنسانية لكل البشر على مر العصور • والمؤسسات التعليمية على اختلاف مستوياتها، بدءاً من المراحل الابتدائية إلى الجامعة، هي جزء رئيس من دعائم ذلك التطور ، اعتماداً على مناهج تعليمية جادة ومكملة لبعضها بعضاً، ومنبثقة عن حاجات المجتمع، بجهود معلمين أكفاء مُدرّبين تدريباً عالياً ومتواصلاً، بما يساهم في تعزبز قدرات الطلاب الذهنية والإبداعية، مع محاولة الربط الدائم بين النظرية والتطبيق، بين النقد البنّاء واحترام وجهات نظر الآخرين، والتمييز بين المصلحة العامة والخاصة، وتغليب الأولى على الثانية، وإنشاء معاهد أو جامعات عربية متطورة، والاستفادة من خبرات الدول التي قطعت أشواطاً طويلة في تلك المجالات. نحو هذه الغاية، أقترح إنشاء جامعات أو معاهد علمية عربية في دول متطورة علمياً وتقنياً على غرار الجامعات الغربية في بعض الدول العربية، مثنلًا إنشاء جامعة بغداد في لندن للعلوم الطبية، أو جامعة دمشق في باربس للعلوم الزراعية وجامعة القاهرة في واثنطن لعلوم الحاسب، إلخ، بحيث يتم التدريس فيها بمناهج مثرجمة إلى العربية من إحدى الجامعات المحلية في بريطانيا أو فرنسا أو أمريكا، على غرار الجامعة الأمريكية في بيروت أو الجامعة الأمريكية في القاهرة أو كثير من الجامعات الأمريكية في دول الخليج، التي تدرس مناهجها باللغة الإنكليزبة في البلدان العربية. وهكذا، يمكن لمنل هذه الجامعات تزوبد الطلاب العرب بالخبرة والمعرفة العلمية المحدثة باستمرار، وبلغتهم من حيث يتم انتاج المعرفة والتطوير التقني والعلمي، مما قد يساهم في رفع المستوى العلمي في البلاد العربية.

يعاني العرب حالياً من تأخر كبير على المستوى العلمي والتقني، على الرغم من الإمكانيات المادية الكبيرة لمعظم الدول العربية، التي أغْرَتْ وتُغري كثير من الجامعات العالمية لفتح فروع فيها، والفوز بعقود مالية 
كبيرة ورسوم تعليم باهظة لا يقدر عليها إلا الأغنياء، وهو ما يجعل الهدف الرئيس لتلك المؤسسات هو الربح أكثر منه نشر العلم والمعرفة [1].

ولمواكبة النطور العلمي، لا بد من مراجعة متأنية لسياسات التعليم في المدراس والجامعات العربية، والاستفادة من خبرات الدول المتقدمة في تللك المجالات لإيجاد حلول مستدامة للمشاكل البيئية والطبية والثقنية، الني تعاني منها معظم الدول العربية.

وفي هذا الإطار، أقترح إنثاء جامعة، أو جامعات، أو معاهد عربية للعلوم في بعض الدول المتطورة على غرار الجامعات الغربية في البلاد العربية، بحيث تكون اللغة العربية لغة التدريس فيها بجانب لغة البلد المضيف، لمواكبة آخر المستجدات العلمية في الطب والعلوم وعلاج الأمراض والتقنيات الحديثة وترجمتها إلى العربية، بما يجعل ويُقي مناهج تللك الجامعات مُحدّثة باستمرار ومتزامنة مع ما يتم انتاجه وتعليمه في البلدان المتقدمة علمياً وتقنياً. ثم يمكن ابتعاث الطلاب العرب المتفوقين للاراسة في مثل هذه الجامعات، لمتابعة تعليمهم العالي والحصول على الخبرة والاطلاع على آخر التطورات العلمية والطبية بلغتهم الأم.

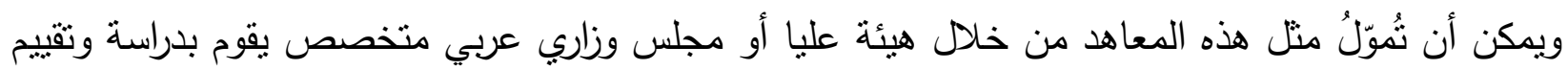
الحاجات العلمية والطبية العربية، والحصول على الموافقات والإجراءات الإدارية، ضمن إطار تعاون علمي هلي وقانوني معترف به من قبل أعلى الهيئات الحكومية لجميع الأطراف المعنية في البلدان العربية والبلاد المستضيفة. ويمكن أيضاً الاقتصار على إنشاء معاهد أو فروع لجامعات عربية في الخارج للعلوم الطبية والزراعية والهندسة الثقنية (مثل علوم الحاسوب)، لأن هذه التخصصات مهمة إنسانباً واقتصادياً وتجارياً.

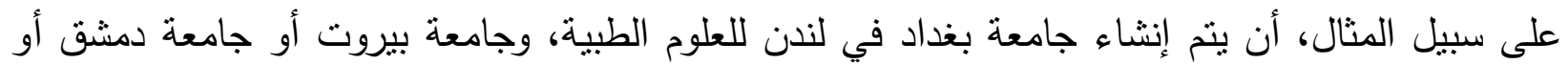

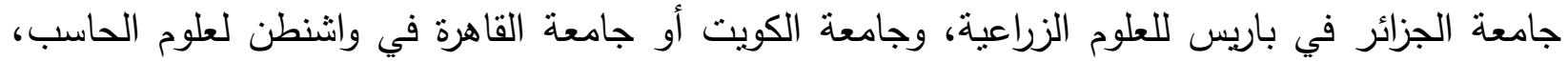

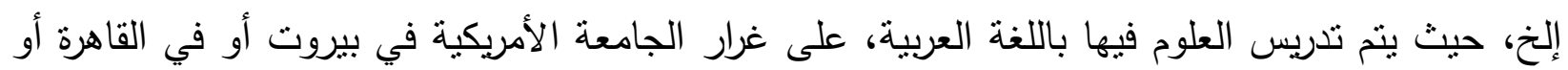
كثثر من الجامعات الأمريكية في دول الخليج، التي تدرس مناهجها باللغة الإنكليزية.

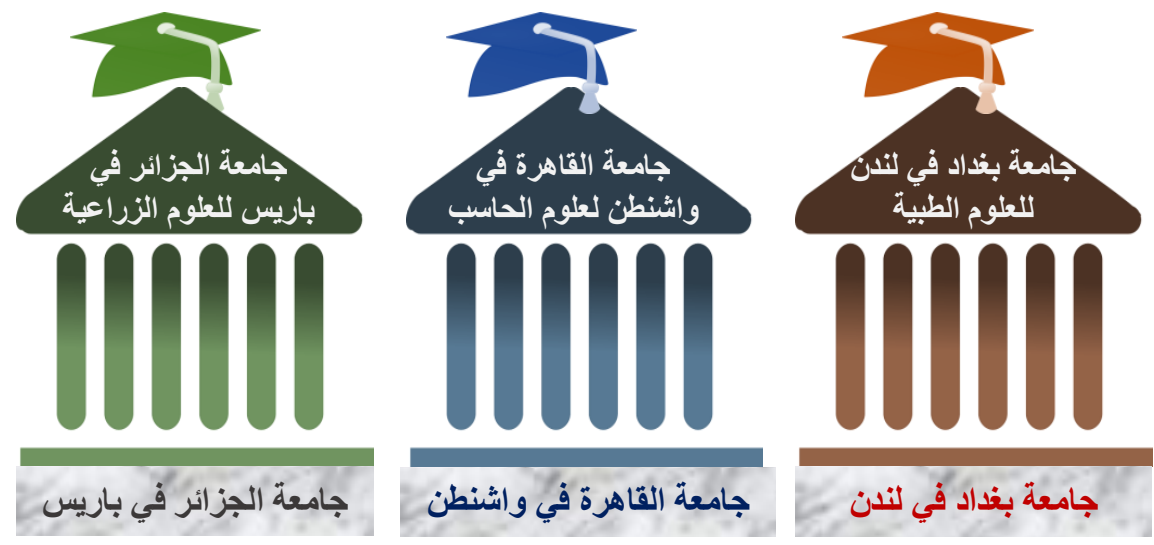


يمكن أن تحقق مثل هذه المشاريع فوائد كثيرة على القطاع العلمي العربي بزيادة روابط التعاون العلمي والتقني

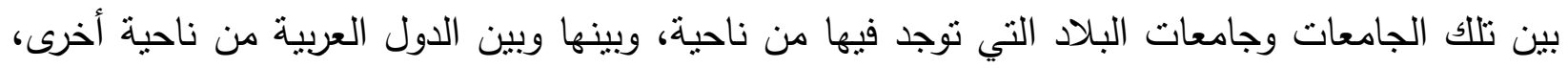
لتخريج طلبة عرب بمناهج علمية متطورة باللغة العربية. ولا أعتقد أن التكاليف الإدارية والقانونية والمالية لإنثاء متل هكذا مؤسسات في الدول المتطورة ستكون أكثر من تكاليف شراء بعض الأندية الرياضية أو أوله القصور الفخمة التي يتسابق إليها بعض العرب الأغنياء، بل يمكن تحويل تللك القصور الفارهة، الفارغة، إلى هلى

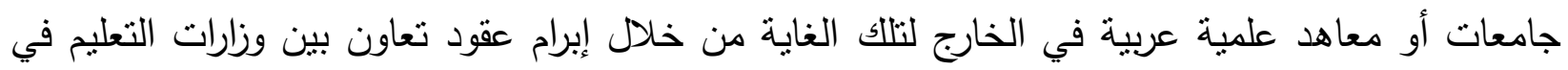
تللك البلدان ونظيراتها في الدول العربية للحصول على تراخيص حكومية رسمية بإنثاء منل هذه الجامعات،

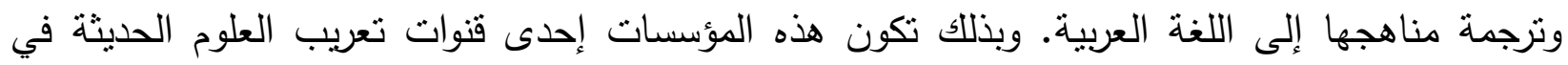
الخارج، ومتابعة آخر المستجدات العلمية من منبعها كإحدى قنوات التواصل المعرفي بين العرب وغيرهم من الأمم المتطورة علمياً وتقنياً. ويمكن أن تكون الدراسة برسوم رمزية جداً، لا ترهق كاهل الطلبة، ولا تجعل تلألك

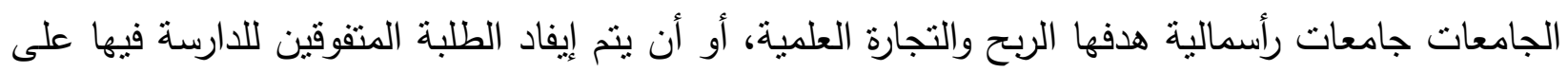
حساب الدول العربية أو الجامعات التي يتخرجون منها في البلدان العربية.

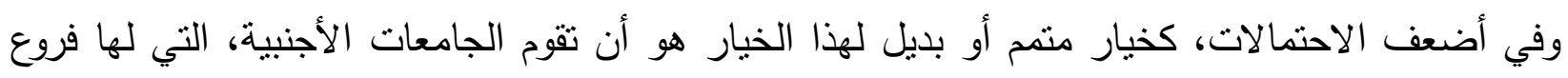

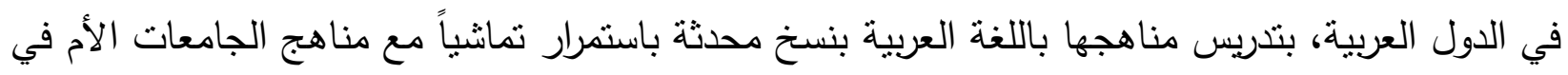

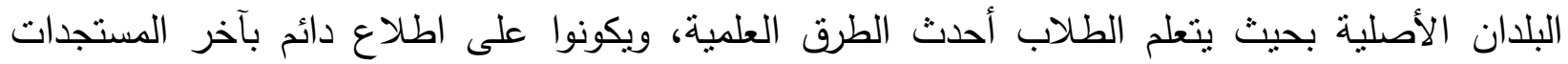
المعرفية والتقنية، كما لو أنهم كانوا يدرسون في الجامعات الأم، التي تنبع لها الفروع الموجودة في البلاد

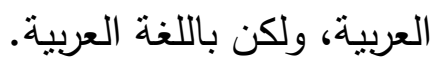

\section{References}

مراجع

1. Moustafa, K., Promoting an academic culture in the Arab world. Avicenna J Med, 2018. 8(3): p. 120-123. 